\title{
XLVI. Experiments on the radiation of heated gases
}

\section{John Evershed}

To cite this article: John Evershed (1895) XLVI. Experiments on the radiation of heated gases, Philosophical Magazine Series 5, 39:240, 460-476, DOI: 10.1080/14786449508620744

To link to this article: http://dx.doi.org/10.1080/14786449508620744

曲 Published online: 08 May 2009.

Submit your article to this journal $\pi$

Џll Article views: 4

Q View related articles $\longleftarrow$

Citing articles: 1 View citing articles 5 
and thus $\mathrm{F}$ can never assume negative values. Also

$$
\mathrm{F}=\mu\left(2 a^{2}+2 b^{2}+2 c^{2}-\frac{2}{3} \theta^{2}+\mathrm{A}^{2}+\mathrm{B}^{2}+\mathrm{C}^{2}\right), .
$$

this is the expression for the "Dissipation Function" F originally given by Lord Rayleigh. The cause for this denomination is obvious. Suppose $a, b, c, \mathrm{~A}, \mathrm{~B}, \mathrm{C}$ to have their sign changed; the sign of $p \theta$ will change but that of $F$ will not; hence the term $p \theta$ corresponds to a reversible, the term $\mathrm{F}$ to an irreversible phenomenon. Indeed the conversion of molar energy into molecular energy which goes on, of whatever kind the existing disturbance may be, is irreversible. We have thus before us an example of Dissipation of Energy in a purely dynamical system.

3 . If we now suppose that every molecule possesses an amount $\mathrm{M} h$ of internal energy in addition to that which depends on its motion of translation, we shall have

$$
\frac{\delta}{\delta t}\left[\frac{1}{2} \rho\left(u^{2}+v^{2}+w^{2}+\overline{\xi^{2}}+\overline{\eta^{2}}+\overline{\zeta^{2}}\right)+\rho \bar{h}\right]=0 . \quad .
$$

Now it is easily seen that $\delta \bar{h} / \delta t$ and $d \bar{h} / d t$ are equivalent, since $\bar{h}$ cannot be affected by external forces any more than by convection; therefore

$$
\begin{aligned}
\frac{1}{2} \rho \frac{d}{d t}\left(\bar{\xi}^{2}+\right. & \eta^{\overline{2}}+\overline{\zeta^{2}}+\rho \frac{d \bar{h}}{d t}+\rho \overline{\xi^{2}} a+\rho \overline{\eta^{2} b}+\rho \overline{\bar{\zeta}^{2}} c+\rho \overline{\eta \bar{\zeta}} \mathrm{A}+\rho \overline{\zeta \xi} \mathrm{B}+\rho \overline{\xi \eta} \mathrm{C} \\
& +\frac{1}{2} \frac{\partial}{\partial x}\left(\rho \overline{\xi^{3}}+\rho \overline{\xi \eta^{2}}+\rho \overline{\xi \zeta^{2}}\right)+\frac{1}{2} \frac{\partial}{\partial y}\left(\rho \overline{\eta \xi^{2}}+\rho \overline{\eta^{3}}+\rho \overline{\eta \zeta^{2}}\right) \\
& +\frac{1}{2} \frac{\partial}{\partial z}\left(\rho \overline{\zeta \xi^{2}}+\rho \overline{\eta^{2}}+\rho \overline{\zeta^{3}}\right)=0 . \quad . . . . . \cdot(31)
\end{aligned}
$$

Maxwell puts $\rho \bar{h}=\frac{1}{2}(\beta-1) \rho\left(\overline{\xi^{2}}+\overline{\eta^{2}}+\overline{\zeta^{2}}\right)$; if this relation is adopted, equation (31). will be that numbered (94) in Maxwell's paper, with a difference of factor, however, in the terms relating to conduction of heat. The foregoing deduction will not be subject to the criticism which M. Poincaré has offered with respect to Maxwell's deduction (see Comptes Rendus, vol. cxvi. p. 1017 ; see also ibidem, p. 1165, where the internal energy of the molecules is taken into account in the same way as that here adopted).

XLVI. Experiments on the Radiation of Heated Gases. By JoHN Evershed*.

\section{[Plate VII.]}

THE renewed interest aroused in certain fundamental 1 questions in spectroscopy by the publication of the researches of Pringsheim, Paschen, and Smithells has led me recently to undertake a series of observations on the visible

* Communicated by the Author. 
radiations of certain elementary substances, particularly iodine and sodium, when their vapours are subjected to external heating. The important article published in the Philosophical Magazine (March 1894), by Prof. Smithells, may be said to have furnished the main incentive in this work, as it likewise determined the direction followed in the experiments.

I am further indebted to Prof. Smithells, to whom I submitted a statement of my results, for valuable advice and criticism, which has enabled me to anticipate certain objections that might have been raised to my conclusions.

The experiments were undertaken primarily with a view to obtaining a closer personal acquaintance with what I may call the "spectroscopic behaviour" of heated gases. I had no idea that they would lead to results which could throw any new light on the questions discussed in Prof. Smithells paper. Nevertheless, after continuing the experiments in a somewhat desultory fashion for some six months, and with very simple appliances, certain facts have presented thenselves in a prominent, way, which, so far as I can discover, have not been sufficiently noticed by previous workers, and which would appeur to lend a strong support to the view there advocated, namely, that the luminosity of gases in flames may be directly due to high temperature, and that external heating is in itself sufficient to make a gas emit visible rays.

The fact that iodine vapour can he easily mado incandescent by external heating was noticed by Salet (Spectroscopie, p. 173); and in the article above mentioned Prof. Smithells concludes that this luminosity can only be due to heat, and that chemical action plays no part in the phenomenon. It seemed, therefore, of interest to determine, first, the character of this indine emission, and then to discover whether the property of glowing by mere heating was peculiar to iodine, or was shared in greater or less degree by other allied elements when vaporized, particnlarly by those which exhibit strong absorptive properties on light. After having thus gained some experience with the less easily oxidizable metalloids, I proposed finally to approach the question of the radiation of heated metallic vapours, to determine whether or not their cbaracteristic spectra can be produced by mere heating.

It will perhaps be worth while at the outset to describe briefly a few simple experiments made to determine whether the jodine glow is affected in any way by the nature of the gas in which it is heated. I will describe them as nearly as possible in the order in which they were performed.

Experiment I.-A little iodine was placed neai' the closed end of a piece of hard glass tube of about $7 \mathrm{~mm}$. bore, the 
other end being open to the atmosphere. This was suspended horizontally, and a Bunsen flame brought under the central part; when about 5 or $6 \mathrm{~cm}$. of the tube had become nearly red-hot, the flame was held for a few seconds under the part containing the iodine, which immediately volatilized, filling the whole tube with coloured vapour. A bright reddish glow then appeared in the heated portion of the tube.

Eap. II.-Iodine was heated in a tube as in I. but closed to the atmosphere, and in which air was replaced by nitrogen. No difference was seen in the emission of light.

Exp. III.-The same as II., excepting that hydrogen was substituted for nitrogen. In this case also the glowing appeared, the same as before, but it could not be maintained as combination took place between the hydrogen and iodine, and the colourless hydriodic acid formed emitted no light.

Exp. IV.-In $\mathrm{CO}_{2}$ the same result was obtained as in nitrogen.

Exp. V.-A thick glass tube containing iodine was exhausted with an air-pump until the pressure fell to about $20 \mathrm{~mm}$. It was then heated so as to expel the remaining air by volatilizing a portion of the iodine, and then sealed off. The whole length of the exhausted tube was next warmed up until the density of the iodine vapour was sufficient to give the usual deep violet colour. On strongly heating a short section of the tube at this stage, the usual reddish-yellow glow appeared exactly as in the other experiments.

These preliminary experiments, therefore, as far as they go, entirely favour the view that the glowing is determined by heat alone, and not by chemical "luminescence." For the phenomenon appears to be quite independent of the gas in which the iodine is heated, and in III., where chemical combination actually occurs, the light, instead of being intensified, is extinguished.

To determine the character of the light, things were arranged as in $\mathrm{I}$., the glowing tube being observed with a spectroscope, having a lens in front to throw an image of the hot part of the tube across the slit of the instrument. Before beating the iodine a faint streaky spectrum, due to glowing opaque particles in the glass, was all that could be seen; but immediately the iodine was vaporized a bright spectrum shone out. This appeared perfectly continuons, and similar to that given by a red-hot iron wire, with which it was directly compared. No sign of resolution into lines could be made out even with a highly dispersive train of five prisms.

Next a ray of white light from the flame of a paraffin lamp was made to pass through the glowing tube and enter the spectroscope, after having suffered absorption by the heated vapour. 
The dark bands, characteristic of cooler iodine vapour, were seen to be unchanged, and there was no sign of any continuous absorption. Thus at the temperature of the experiment iodine emits a continuous spectrum, and does not emit only those rays which it absorbs.

The question whether iodine is the only element having this property of glowing with a continuous spectrum at comparatively low temperatures was next investigated. A series of experiments with the allied element bromine immediately showed that this was not so, for the vapour of this substance was also found to glow brightly under similar circumstances.

The bromine glow seems indeed to be quite as conspicuous as that of iodine: the spectrum also was found to be perfectly continuous.

It was then thought that chlorine, being also a coloured gas and allied to the others, would probably be found to emit light in the same way. Accordingly arrangements were made for generating and thoroughly drying this gas and heating it in a glass tube, as in the foregoing experiments. The first attempts to see the glow were made, as before, from outside the tabe; but they led to negative, or, at the most, very doubtful results. A very slight alteration in the mode of viewing the heated gas soon, however, revealed a distinct and unmistakable, though faint, luminosity. The experiment was arranged in the following way :-Chlorine from a generating flask, after being freed from $\mathrm{HCl}$ and dried by passing slowly through a long tube of calcium chloride, was led into a straight piece of combustion-tube of about $7 \mathrm{~mm}$. bore, heated strongly in the middle. This tube connected on to a larger glass tube containing a total-reflexion prism placed in line with the central axis of the heated tube : this enabled one to observe the heated gas " end on" without the interposition of the red-hot glass, and a dark background was obtained by covering up the further end of the combustiontube with opaque material.

In this way observations were made, first, with common air only passed through the apparatus, and afterwards with dried chlorine, about $5 \mathrm{~cm}$. of the tube being kept meanwhile at the highest temperature attainable with an ordinary Bunsen burner. With air, the centre of the tube remained perfectly dark, and no trace of glowing solid matter in the form of dust could be seen. With chlorine, on the other hand, a faint greenish glow gradually filled up the previously dark central bore of the tube, appearing first on the lower or hottest part. The light, although brighter on the lower side, appeared perfectly uniform in texture so to speak, unlike the glow produced by incandescent solid particles, which can be seen 
under certain circumstances as wreaths and streaky clouds moving with the convection-currents in the tube *.

Being satisfied by repeated trials that the glow was really due to incandescent chlorine, an endeavour was made to determine the spectroscopic character of the light. It seemed almost hopeless to observe the spectrum directly, although with a spectroscope of very low dispersion and wide slit the spectrum appeared to be continuous; but it could only be traced between the positions of $\mathrm{D}$ and $\mathrm{F}$ of the solar spectrum, probably owing to the greater sensitiveness of the eye to that region. An indirect method was, however, devised. The depth of the layer of cool chlorine traversed by the light from the hot gas was, in the first instance, about $20 \mathrm{~cm}$., that being the distance between the prism and the hot part of the tube. The absorbing effect of this thickness of gas was tested by varying the distance, heating the tube successively at 15 and $30 \mathrm{~cm}$. from the prism. If the spectrum of the light emitted consisted of lines or bands corresponding to the absorption-lines of chlorine, there should be a marked increase of absorption after traversing $30 \mathrm{~cm}$. as compared with $15 \mathrm{~cm}$. But no difference was perceptible in the intensity of the glow whatever the distance traversed, showing that the cool gas exercises very little absorption on the light coming from the hot.

The inference, therefore, is that chlorine, like iodine and bromine, emits other rays than those absorbed, and probably shines with continuous light; the selective absorption of the cool gas merely giving the glow a greenish tint.

There seems no reason to doubt that the luminosity of chlorine, as of its allied elements, is directly due to the heating, and that chemical changes are not concerned in the phenomenon.

The Sulphur Group. - Similar results have been obtained with sulphur and selenium. The glowing may be observed either "end on" in a red-hot porcelain tube filled with hydrogen or nitrogen, or the element may be simply sealed in a hard glass tube in air. If a small portion of the tube be then heated strongly, a faint glow can be seen while distillation is going on, and the heated space is filled with vapour.

Phosphorus.-Experiments with this substance have so far led to negative results. After all the phosphorescence due to traces of oxygen has disappeared from the tube, the vapour appears to give no light.

* The glowing of heated chlorine may be more conveniently observed in a porcelain tube connected with a T-tube having a glass plate cemented in one end of the $T$. If the Bunsen is concentrated on the tube by means of a fire-clay arch placed over it, the glow uniformly illuminates the bore of the tube. 
Arsenic.-The vapour of this substance glows distinctly when heated in nitrogen. In hydrogen also the glow is distinet, but fainter than in nitrogen.

To sum up, then, it appears that besides iodine, the vapours of bromine, chlorine, sulphur, selenium, and arsenic can all be made more or less incandescent by heating to the temperature at which glass combustion-tube softens, and the light emitted by each of these glowing vapours appears to give a perfectly continuous spectrum; whilst the corresponding absorption spectra are selective. Thus there is no such close relation between emission and absorption as is implied by Kirchhoff's law of radiating bodies. There seems, however, to be a general relation between the total absorbing and radiating power for the visible rays : those vapours which are highly coloured and absorb strongly in the visible spectrum also radiate conspicuously in that part of the spectrum, whilst colourless non-absorbing vapours, such as phosphorus, emit no perceptible light when heated.

That the glowing in these cases in no way differs from the glowing of heated solids seems, to say the least, extremely probable, for there is no evidence whatever that chemical changes accompany the luminosity; and there is besides the fact that when direct combination does occur between the vapour and the gas in which it is heated, as in the case of iodine in hydrogen, and possibly also arsenic in hydrogen, there is no luminous effect at all.

It may be questioned, however, whether molecular dissociation may not be concerned in the radiation, or alternate dissociation and reaggregation of the atoms of the molecules. For, according to the kinetic theory, at a given temperature and pressure the vapours may contain a certain proportion of free atoms distributed among the more complex molecular groups, but the individuality of these uncombined atoms will continually change whilst the proportion remains the same, for there will be a constant reaction or interchange going on between the atoms and the molecules. The emission of light may be supposed to depend on this act of union or disinion of the atoms, the radiant energy being indirectly derived from the heat supplied to the system to maintain the temperature.

Thus in the case of the diatomic gases iodine, bromine, and chlorine, a proportion of the molecules $\mathrm{I}_{2}, \mathrm{Br}_{2}, \mathrm{Cl}_{2}$ may dissociate into $2 \mathrm{I}, 2 \mathrm{Br}, 2 \mathrm{Cl}$, and sulphur vapour may similarly dissociate from $\mathrm{S}_{6}$ to $3 \mathrm{~S}_{2}$, and so on. From recent determinations of the vapour densities of the halogens, it appears that iodine begins to dissociate between $600^{\circ}$ and $700^{\circ} \mathrm{C}$., at a pressure of 1 atmosphere*. Chlorine, on the other hand, * Crafts and Meier, Ber. deut. chem. Ges. xiii. 
remains at a normal density corresponding to $\mathrm{Cl}_{2}$ between about $200^{\circ}$ and $1200^{\circ} \mathrm{C} .{ }^{*}$ With regard to the former element, the temperature at which dissociation commences (say $600^{\circ}$ ) is not much above that at which the glowing is first seen, and as in most of the experiments the iodine or bromine vapour is largely diluted with a neutral gas, so that the partial pressure is a good deal less than one atmosphere, it might well be supposed that dissociation was going on even at the lowest temperature at which the glow can be seen. But in the case of chlorine dissociation begins at some $500^{\circ}$ above the temperature of my experiments (assumed at about $700^{\circ}$ ) : moreover, there is no dilution of gas, which is observed at the atmospheric pressure, so there can be no question of dissociation here ; or at any rate, as there is no independent evidence of it, we have no more right to assume it as a cause of the luminosity than we have in the case of glowing solids.

But, apart from the fact that chlorine can be made incandescent although it is not dissociating, it appears to me that the general relation mentioned above between radiation and absorption of the visible rays, and the fact that the intensity of the glowing of the more absorptive vapours (the others being too difficult to observe) appears to closely follow that of a solid raised simultaneously through the same range of temperature $\dagger$, gives strong support to the view that there is no essential difference between gases and solids in the manner in which they radiate, at any rate under the conditions of the foregoing experiments. If dissociation were concerned, say, in the case of glowing iodine, one would expect the intensity of the light to rapidly increase when the temperature is made to approach the actual temperature of dissociation, where the maximum interaction of the atoms occurs. It should in fact increase in a much greater ratio than in the case of a glowing solid. But I have failed to detect any evidence of such relative increase on the part of either iodine or any other glowing gas. Further, a decrease of density (by exhaustion or dilution) will facilitate dissociation, and thus should tend to counteract the reduction of luminosity due to a smaller number of molecules concerned. But no such effect is in fact to be seen under these conditions.

* J. M. Crafts, ibid. xvi.; also Jahn, ibid. xv.

+ The radiation from iodine may be ensily compared with that of a solid at the same temperature, by placing a small piece of carbon inside the heated portion of the glass tube described in exp. I. Also when the glass contains opaque particles, these are soen to glow with the same intensity as the lodine, whatever the temperature, when the vapour is of sufficient density to give the maximum luminosity. 


\section{The Sodium Radiation.}

Having thus far failed to produce discontinuous spectra by external heating, I next tried what could be done with metallic vapours. Sodium was the metal chosen for the initial experiments, the powerful absorption produced by the vapour of this element on " D" light seeming, on Kirchhoff"s hypothesis, to give the best chance of success at the very moderate temperatures I could command with a single large Bunsen flame.

The form of apparatus used in the earlier experiments was designed with a view to excluding, as far as possible, from the tube in which the sodium was to be heated any gaseous substances that might be expected to react chemically with the vapour of the metal : the emission phenomena produced under these conditions being then compared with that produced when traces of oxygen or moisture were purposely allowed to remain in the neutral gas in which the sodium was volatilized. In the diagram (Plate VII. fig. 1) $\mathrm{A}$ and $\mathrm{B}$ are two similar gas-holders; a rubber tube leads from A to a couple of wash-bottles $\mathbb{S}^{\prime}, \mathbb{S}^{\prime \prime}$, containing strong sulphuric acid; from $\mathrm{S}^{\prime \prime}$ a long tube of hard glass, $\mathrm{P}$, containing a little phosphorus leads into the drying-tube $\mathrm{C}$, which is packed with calcium oxide and calcium chloride-the former to remove carbonic acid, and the latter traces of water which may remain in the gas used after passing the sulphuric-acid bottles. The drying-tube connects on to the porcelain heatingtube $\mathrm{H}$ through a metal $\mathrm{T}$-piece, one end of the $\mathrm{T}$ having a glass plate carefully cemented in so that one may look along the inside of the heating-tube, to which the $\mathrm{T}$ is connected by rubber tube tightened with wire, both connexions being also buried in sealing-wax. At the other end of $\mathrm{H}$, which is covered in the centre by a fireclay arch, is a second $T$ of glass, one limb connecting with a glass gland or stuffing-box, $G$, with pierced rubber ends, and filled with mercury. A long steel rod passes through the gland, the end being flattened to a spoon-shape; this can be pushed along to the centre of $\mathrm{H}$, or drawn out past the entrance of the side tube of the $T$. This side tube is closed by a perforated rubber stopper, through which a small glass tube passes bearing a small reflecting prism cemented to the end, which is thus closed up; but in order to allow of the escape of the gases, so that a current may be set up in the apparatus, a hole is blown in the side of this tube near the prism. The outer end of the tube is connected by rubber tubing to another wash-bottle $\mathrm{S}^{\prime \prime \prime}$ containing sulphuric acid, and from this again a tube leads to the gasholder B. Thus the entire apparatus forms a closed circuit and has no inlet or outlet. The gas-holders have each a 
Phit.Mag. S. 5. Vol 39. Pi. Yit.

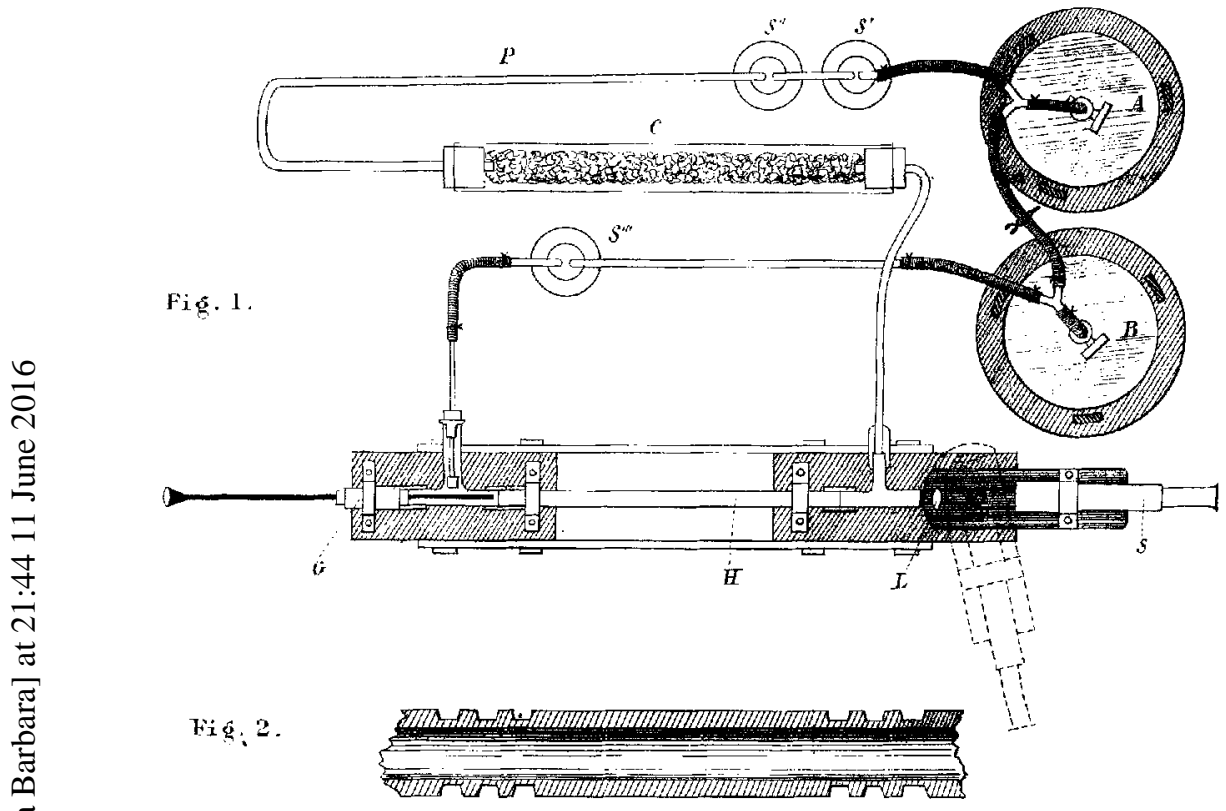

EMISSION
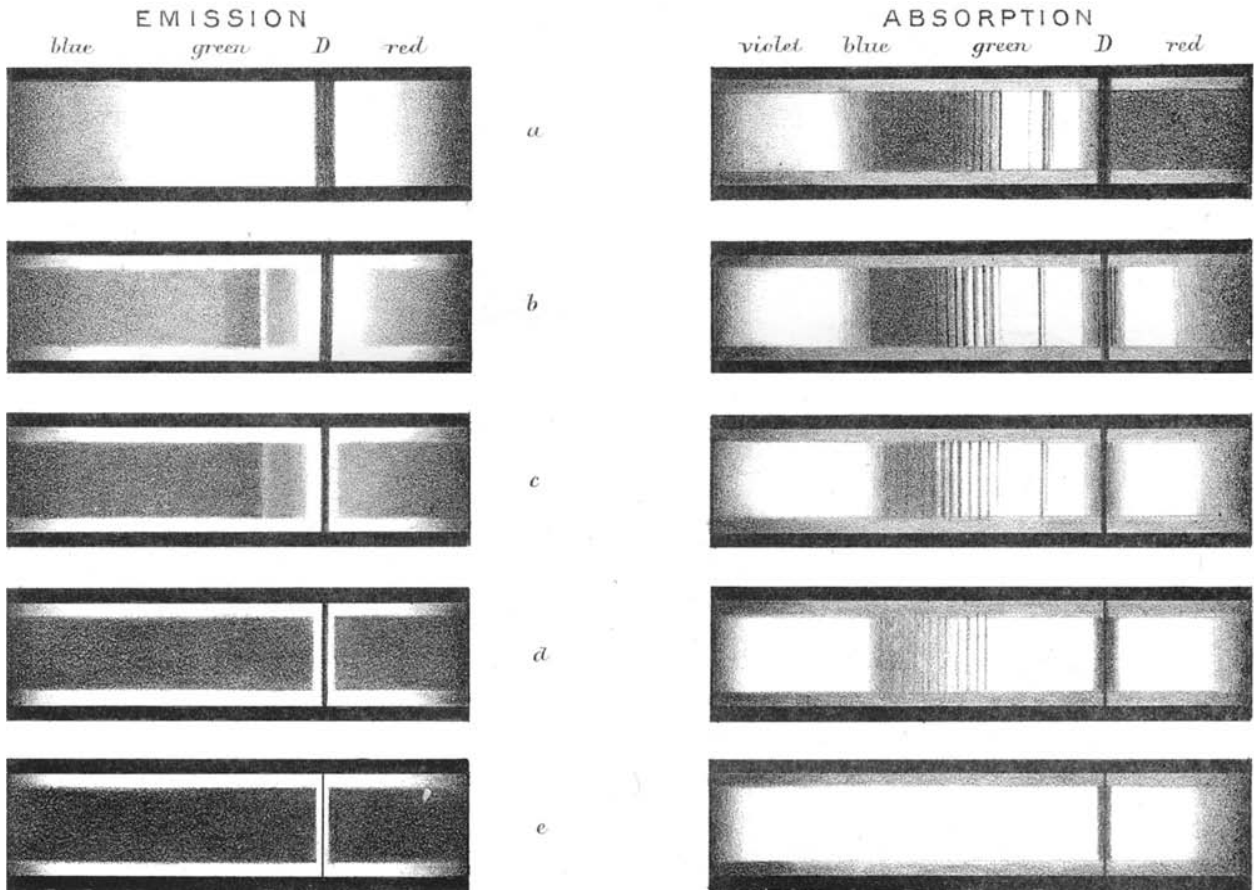

Fi \&. 3 .

$F$ ig. 4

Wote. - The comtinuous spedrum at the ediges comes from the glowing walls of the tube Twintern Brus lith. 
Y-tube attached, one branch of the $\mathrm{Y}$ leading to the apparatus and the other connecting $A$ with $B$ by means of a rubber tube carrying a clip. The $\mathrm{S}$ at the foot of the diagram is a small direct-vision spectroscope, and $\mathrm{L}$ is a lens focussing the central parts of the tube $\mathrm{H}$ on to the slit of the instrument; both are attached to a strip of hard wood movable horizontally about an axis placed between the lens and the slit. This enables one to instantly push aside the spectroscope into the position shown by dotted lines and observe the glowing tube directly.

To observe the sodium-spectrum, one fills the gas-holder A with some indifferent gas containing no oxygen, or only a trace of that element, such as nitrogen or hydrogen, or ordinary coal-gas. When full it is disconnected with the gas-generator or gas-main, as the case may be, and.connexion is made with the apparatus. Next, weights are put on A until sufficient pressure is obtained to drive a current of gas through the wash-bottles, drying-tube, \&c. into B. Then the Bunsen is lighted under the porcelain tube, which it presently heats up to a bright incandescence for about two inches of its length. After sufficient dry gas has passed through, and all trace of moisture has gone, the current is stopped by closing the stopcock on A, and a small pellet of sodium is dropped into the steel spoon through the side tube of the glass $\mathrm{T}$, the stopper with the inner tube and prism being removed for this purpose and quickly replaced. The current is then restarted, to drive away any oxygen that may have diffused in by the operation, and at the same time the tube $\mathrm{P}$ is gently heated by a spirit-lamp flame until a small faintly luminous flame is seen, indicating combination of the last traces of oxygen with the phosphorus. After this has gone on a sufficient length of time, and the apparatus may be considered to be free from oxygen, water, and carbonic acid, the stopcock on A is again closed and the steel spoon carrying the sodium is pushed into the hot part of the tube $\mathrm{H}$, turned over, the sodium shaken out, and the spoon again withdrawn past the entrance of the side tube. Now the tube carrying the prism is pushed down into line with the tube $\mathrm{H}$, and the white flame of a paraffin-lamp is placed close alongside the glass $\mathrm{T}$, so that a ray of white light can be made to traverse the glowing vapour in $\mathrm{H}$. One may now observe at will the absorption or emission spectrum of the glowing sodium by the simple operation of turning the lamp-flame up or down.

The experiments actually performed with this apparatus may be thus briefly described :-

I. With the porcelain tube strongly heated, a slow current of coal-gas, not specially freed from oxygen, was allowed to 
circulate in the apparatus, no sodium being admitted. A distinet and fine sodium line was visible in the spectroscope, which increased in brightness when a little air was mixed with the gas, but which gradually faded to invisibility when the phosphorus tube was heated so as to eliminate oxygen.

The explanation of this result appears to be simple enough. The trace of oxygen remaining in the coal-gas combines with the hydrogen when it reaches the hot part of the tube, and the "flame" so formed (which, however, is not visible as such, except when a large quantity of $O$ is present) becomes tinted by the salts of sodium, which in excessively minute quantity are known to be driven off from the porcelain at a red heat, just in the same way as the Bunsen flame outside is tinted. This fine double $\mathrm{D}$ line, therefore, may not be the result of heat alone, since it is developed as a consequence of chemical reactions.

II. A pellet of clean sodium was placed in the steel spoon, and the gas-coal-gas-allowed to circulate, the phosphorus being heated. The line seen in Experiment I. was watched, and some time after it had quite disappeared the current was stopped and the sodium pushed into the hot part of the tube. Instantly the central bore of the porcelain was filled with light, which in the spectroscope was found to be perfectly continuous, but crossed by a very wide black line at $D$. Gradually the continuous spectrum faded, and as it became fainter the dark D line was seen to be bordered with a fringe of light on each side; and as the vapour became less dense, owing to the distillation of the sodium into cooler parts of the tube, the $\mathrm{D}$ line went through the changes represented in fig. 3 , in the order $a, b, c, d$, finally persisting as a rather wide bright line in which a very fine dark line could usually be made out*. But at any stage of the experiment, the dark central line could easily be extinguished by allowing a gentle current of gas to push back the cooler absorbing layer into the hotter regions. Now the question to be decided was whether this broad bright, hazy D line was or was not the result of chemical activity.

III. In this experiment the phosphorus tube and the drying-tube were cut out of the circuit, the gas-holder A being connected directly with the heating-tube. With the current of gas stopped, the $D$ line appeared as in the last experiment, but observations were somewhat impeded by opaque clouds of oxide which hung about the cooler parts

* The dispersion of the spectroscope employed in all the sodium experiments being insufficient to separate the two components of the $D$ line, it is evident that when this line appeared widened the two were really fused into one broad band.

Phil. Mag. S. 5. Vol. 39. No. 240. May 1895. 2 I 
of the tube. When, however, a slow current of the undried gas was allowed to impinge on the sodium vapour, by partly opening the stopcock $A$, the absorption-line vanished and an intensely brilliant but fine line appeared in its place in the centre of the broad but relatively faint emission-line. This sharply-defined narrow line resembled the ordinary D line seen in flames. It was brighter than the continuous spectrum of the glowing sides of the tube, on which it appeared to be superposed.

IV. Atmospheric nitrogen was substituted for coal-gas in the gas-holders, and was freed from traces of oxygen and dried before entering the heating-tube by passing over heated phosphorus and through a tube of $\mathrm{CaCl}_{2}$. The phenomena observed on volatilizing the sodium in this gas were in every way the same as in coal-gas. It was subsequently found that the same results could be obtained with unpurified nitrogen, or even with common air, the sodium itself effecting the purification almost immediately on vaporizing, produciug at the same time a brilliant flash-in the spectroscope a brilliant but sharply-defined and narrow D line-and clouds of oxide; afterwards showing the broad hazy emission-line and the central black absorption-line, when all the oxygen in the tube had been consumed and the oxide had subsided.

These results appear to me to show that impurities in the neutral gases used are not concerned in the production of the broad hazy emission-line, for when traces of these, particularly oxygen and moisture, are known to be present and are allowed to impinge on the sodium vapour, a line is seen which is fine and sharp, showing that the region of chemical action is only a surface-layer of no great density, whilst the fainter but broad and diffuse $\mathrm{D}$ line, always seen when the vapour is undisturbed, evidently originates at a great depth where the vapour-density is considerable and in a region protected from chemical action by the outer relatively cool layers giving the absorption-line.

While this central region of the vapour may be considered to be well protected by the outer layers from impurities in the neutral gas employed, there still remains the possibility. that the porcelain tube itself reacts with the sodium throughout its heated part, thus furnishing a contintal supply of chemical energy; and some support is given to this view of the case from the fact that the bright line cannot be maintained as a wide line indefinitely without a continual addition of fresh sodium, also the tube becomes much corroded, black silicon being deposited inside : thus proving a reaction between the silicates of the porcelain and the sodium.

In the experiments which follow, the effect of such reactions between the tube and the sodium is eliminated by the use of 
iron tubes in place of porcelain. The first trials were made with a short piece of iron tube about $6 \mathrm{~cm}$. long and $8 \mathrm{~mm}$. bore, bevelled at the ends and fitted between two hard glass tubes of the same diameter, the joints being ground to fit. This made good joints when the iron became red-hot and the glass in contact with it was softened and pressed up tight; but although satisfactory results were obtained in two or three experiments, constant trouble was experienced in the cracking of the glass while cooling. Finally the glass was discarded and a long iron tube prepared (a piece of ordinary $\frac{1}{4}$-inch hydraulic tube). In order to diminish as far as possible the loss of heat by conduction, so as to maintain a high temperature for about $8 \mathrm{~cm}$. in the central part, a number of deep necks were cut in the metal, as shown in fig. 2 , and around these necks a thick ring of asbestos-packing was wound and a fire-clay arch placed over all. Thus the Bunsen flame could be concentrated entirely on the central piece of the tube and the temperature could be maintained inside the tube above the fusing-point of fluor-spar and aluminium, but not reaching that of silver. The rubber connexions between the ends of the iron tube and the two T-pieces gave trouble at first, but subsequently it was found that when buried in a thick layer of plaster of Paris they were completely protected from destruction by heat, the large surface afforded by the plaster of Paris forming an effecturl radiator and preventing the ends from becoming too hot.

Experiment V.-With the iron tube at a bright red heat, the sodium was tipped out from the steel spoon as before, in an atmosphere of carefully dried coal-gas. Now, if in the previous experiments the $\mathrm{D}$ radiation was due to chemical action taking place between the sodium and the oxygen compounds of the porcelain, one ought in this experiment to find, if not an entire suppression of the bright line, at least a striking difference in the radiation. No such difference was, however, to be observed, the intensity remaining precisely the same as before and the sequence of phenomena closely resembling that shown in fig. 3 . The various phases there shown were, however, prolonged almost indefinitely in time, as the sodium never became used up as before, and the distillation into cooler parts of the tube proceeded so slowly that it was necessary to allow a gentle current of gas to drive away the denser vapour giving a continuous spectrum before the $\mathrm{D}$ line itself could be studied. Also throughout the experiment the dark absorption-line was more intense than in porcelain, and it could easily be observed after six hours of continuous heating, when even the emission-line had bscome relatively narrow. This naturally follows from the 
consideration that in the iron the temperature-gradient on each side of the central red-hot part of the tube is much less steep than is the case when porcelain or glass is used, and consequently there is a much greater thickness of relatively cool vapour through which the emission-line is seen.

Under the conditions of these experiments, therefore, the bright $D$ line appears to be quite uninfluenced either by the nature of the neutral gases used and the impurities they may contain, or by the material of which the heating-tube is composed: iron giving exactly the same results as porcelain. It would hardly be safe, however, at this stage of the inquiry to infer that chemical reactions are not concerned in the production of the light; for it would be argued that, as iron becomes slightly porous at a red heat, oxygen, or at any rate some of the gaseous constituents of the Bunsen flame, might find their way into the tube by diffusion from outside, and in this way maintain a continual reaction with the sodium vapour.

In order to diminish the possibility of this diffusion inwards affecting the results, a constant pressure of a few millimetres above atmospheric pressure is maintained within the tube, and if gases diffuse in at all it must be in opposition to the outward diffusing hydrogen. It has been pointed out to me, however, by Prof. Sinithells that in dealing with the D line we are dealing with a reaction that is sensitive to $\frac{1}{180,000,000}$ of a grain of sodium. It is only necessary to suppose, therefore, an equivalent amount of oxygen or other reacting body to be continually present in the tube to determine the $D$ radiation.

While I am not prepared to deny the possibility of such minute traces of oxygen or other bodies constantly finding their way into the middle of the sodium vapour, I consider that any reactions so caused could under no circumstances produce the broad ill-defined line actually observed. At the most a fine double $D$ line would be seen similar to that of a flame tinted with a salt of sodium, where the density of the reacting molecules is not great. Moreover, if reacting bodies were diffusing in from outside the tube-the absorbing layer of the sodium vapour itself forming an effectual barrier in other directions - the action would be greatest in an annular region in contact with the sides of the tube where the incoming molecules first encountered the sodium. This should cause a brightening or widening of the $D$ line at each end *. But there is no such inequality seen : the line is quite uniform in width and brightness throughout its length; showing that if chemical reactions are producing the light, the reacting

* The $D$ line with the optical arrangement employed represents a section of the space inside the hot part of tise tube. 
molecules mnst be uniformly distributed throughout the mass of vapour.

The most telling argument, however, and one which, taken alone, appears to me to prove beyond a doubt that the $\mathrm{D}$ radiation under these conditions is the direct result of the heating, is that derived from a comparison between the emission and absorption spectra.

To effect this comparison, the lamp and reflecling prism previously described are brought into operation, and a beam of white light is made to traverse one side of the heated tube, so that one portion of the slit of the spectroscope is illuminated by transmitted white light, whilst another contiguous portion is illuminated by the $\mathbf{D}$ radiation alone. Under these circumstances the absorption and emission spectra appear side by side in the field of view, and may be readily compared.

Figs. 3 and 4 show the corresponding phases of these spectra (denoted by letters of the alphabet). It will be noticed that the emission-line or band in $e, d$, and $e$ is represented as of the same width as the absorption-band. Careful observation under various conditions as to density shows that, excepting for the dark line in the centre, the bright $\mathrm{D}$ line is in every respect the exact counterpart of the absorption-line, whether the broad hazy band of the dense vapour is siudied, or the relatively narrow line seen with more attenuated vapour.

Assuming, then, that the width of both absorption and emission lines is determined by the molecular density of the absorbing and emitting vapour, it follows that in the densest region every molecule that is concerned in the absorption is also concerned in the radiation. In other words, practically every molecule in the hot part of the tube contrihutes its share to the radiation. But it is surely impossible to surpose that every molecule, or even a large proportion of the molecules, is continually undergoing chemical change. The supply of oxygen or other reacting bodies-supposing they do gain access to the sodium-will never be equal to even a small fraction of the demand; also, if oxidation is proceeding, one would expect to find traces of oxide forming after several hours. But the heating may be continued for six hours at the least without touching the apparatns, and at the end of this time the $\mathrm{D}$ line, with its central absorption-line, is seen as clearly as at the beginning, there being no trace of any opaque clouds of oxide such as are always seen when traces of oxygen are known to be present in the tube.

There seems no possible alternative, therefore, to the obvious and simple explanation which ascribes the radiation to heat alone. If it were assumed that there exists diffused throughout the sodium vapour some substance capable of setting up 
chemical reactions, it might indeed be imagined that a kind of cyclical process of alternate combination and dissociation takes place, the energy supplying the radiation being derived indirectly from the heated walls of the tube: these alternate changes being determined by differences of temperature in the reacting molecules, the cooler combining and the hotter dissociating, or vice versâ. But it is practically certain that under the conditions of these experiments there must always be a large excess of sodium molecules over any others likely to produce such reactions, unless, indeed, hydrogen or nitrogen were to behave in this way towards sodium, and the spectroscopic evidence, as just explained, implies that all the freo sodium molecules are concerned in the radiation.

Or perhaps it may be further argued in support of the "chemical" origin of the radiation, that the sodium molecule is itself undergoing alternate dissociation and recombination. The reasons alieady given against this view in the case of iodine apply even more forcibly in this instance. Thus, if the radiation were due to such action, the intensity should follow the curve representing the change of relative vapour-density with temperature, rising to a maximum at the turning-point in this curve-indicating the greatest interaction between the atoms and the molecules-but falling away to zero when the point of complete dissociation is reached. Now the most. reliable recent determinations of the vapour-density of sodium indicate that at about the temperature of melting cast-iron the vapour is entirely monatomic * : therefore, if we assume? that at the lowest temperature of my experiments the atoms are more or less aggregaled, the relative intensity of the $\mathrm{D}$ line, compared with the continuous spectrum of the glowing sides of the tube, should change as the temperature is increased,-it should get brighter or fainter necording as the actual temperature of dissociation is above or below the initial temperature of my experiments; and it should cease altogether if the temperature be raised to the point where the vapour becomes entirely monatomic.

But as a matter of fact there is no such change of relative intensity. . As the temperature is increased from the point where the radiation begins to be seen, the $\mathrm{D}$ line follows strictly the continuous spectrum of the glowing tube : from the lowest to the highest temperature (a range of some 300 C?. degrees), the gaseous radiation increases in intensity exactly in correspondence with the radiation from the solid, alrsays keeping the same intensity (so far as the eye can judge) as the spectrum of the glowing tube.

* A. Scott, Proc. Roy. Soc. Edinb. xiv. p. 410. 


\section{Origin of the Continuous Spectrum.}

There remains to be considered the continuous light seen when the sodium is first volatilized, and before the broad $\mathrm{D}$ emission-line appears (fig. $3, a$ ). After a number of experiments, made under various conditions as to the purity of the coal-gas or nitrogen employed and density of the sodium vapour, $\mathrm{I}$ conclude that this radiation is really due to free sodium, and that it always appears when the vapour is above a certain density. It is well known that dense sodium vapour gives a remarkable banded absorption-spectrum*. In the experiment alluded to, I have studied the continuous emissionspectrum in relation to this banded absorption, and find that they are intimately connected : thus, when the sodium is first volittilized and a bright glow fills the tube, the vapour appears a splendid violet colour by transmitted light; it is in fact opaque to all rays except the violet; gradually, however, green rays begin to be transmitted (fig. 4, a), then red (fig. 4, $b$ ); the dark space between the green and the violet is now seen to be made up of a large number of black lines (close together with the low dispersion employed), these rapidly decrease in intensity as the vapour diffuses along the tube and becomes less dense, while the black band blotting out the yellow assumes the usual appearance of the wide $D$ absorption-band $\dagger$. The continuous emission glow persists through these progressive changes in the absorption, but becomes gradually fainter, and as the last traces of the absorption-lines in the blue disappear it fades away almost entirely, lenving behind, so to speak, the broad bright emission-band at $D$, the exact reverse of the absorption-band, except for the black line filling up the centre, due to relatively cool vapour in the nearer part of the tube.

It seems, therefore, that dense sodium vapour, like iodine and other coloured vapours giving banded absorption-spectra, emits when in this state light of all wave-lengths, and that the change to discontinuous emission is determined by a reduction of density. There are indications, however, that at higher temperatures the continuous light would give place to bands corresponding with the absorption-bands, just as iodine is said to give a banded emission spectrum when heated

* Vide Roscoe \& Schuster, Proc. Roy Soc. xxii. p. 362.

$\dagger$ A curious phenomenon may be seen when a current or gust of gas is allowed to drive the dense vapour along the tube. The colour of the vapour seen by transmitted light suddenly changes from green to a splendid ruby-red. This is not due to a change in the character of the absorption-spectrum, but alay be explained as an effect of refraction: the sodism vapour, being blown out more in the centre than along the sides of the tube, acts like a prism, refracting the blus and gree 2 rays radially from the centre, the rod alone $\rightarrow b$ sing le sst deviated-passing out at the end of the tube. This effect is more obvious with hydrogen than with nitrogen. 
externally to a high temperature (Salét, Traité sur Spectroscopie, p. 174)* . For if the continuous light is watched at the highest temperature attainable with this apparatus, it is seen not to fade equally in all parts of the spectrum, - a stage is reached (see fig. $3, b$ ) when, in addition to the D emissionline, a green line appears in the place of a conspicuous absorption-line, and a faint glow remains in the blue corresponding to the absorption-bands in that region (figs. 3-4, $b$ ).

\section{Intelligence and Miscellaneous Articles.}

\section{ON THE ROTATIONAL COEFFICIENTS OF THERMAL OONDUCTIVITY}

IN CRYSTALS. BY M. CHARLES SORET.

The method used has been pointed out in a previous note (Archives de Genève, 1893, xxix. p. 355).

Heating by the method of Jannettaz a point on a face not perpendicular to the axis of rotation in a crystal in which the coefficients in question are not zero, isotherms should be obtained which are not symmetrical in reference to that diameter which coincides with the projection of the axis of rotation.

The crystals were mounted and centred at the end of a vertical axis provided with screw motions for setting the face horizontal. A small platinum sphere heated by an electrical current is applied against this face at a point $A$ on the prolongation of the vertical axis.

Isotherms were obtained in the usual way, taking care to turn the crystal steadily and regularly during the heating so that this was symmetrical about the point $A$. This point, the intersection of the face investigated and the axis of rotation, was thus without any possible error in the centre of heating.

After obtaining the isotherm it was examined, without touching the adjustment, by means of an eyepiece with a micrometric scale. The crystal being first placed with its rotational axis in the plane of incidence, a rotation of $180^{\circ}$ about $A$ should modify the points of intersection of the micrometer and the isotherm, if this was symmetrical as regards the centre of heating.

Now no appreciable disymmetry could be observed any more than by the other methods previously employed. Small variations, evidently accidental, alter the direction from one observation to another on the same crystal, amounting to $\frac{1}{30}$ to $\frac{1}{50}$ of the diameter of the curves. Irregularities of the same order were observed on isotropic plates and on faces of crystals, in which no constant deformation of the isotherms could be foreseen.

The researches were made on crystals of dolomite of Binn (face of the prism), and of Traverselle (face of the rbombohedron): on crystals of erythrite and on apatite of the Pfitschthal (face of the prism). The existence of coefficients of rotation in the crystals appears therefore more and more improbable.-From the Archives de Geneve, communicated by the Author.

* If iodine is heated in a hard glass tube in a furnace until the glass begins to fuse, the colour of the glowing vapour changes from yellow to pale greenish white. This probably indicates the change to a discontinuous emission. 